\title{
Dupuytren contracture - how fibromatosis remodels the palmar subcutaneous tissue and its fibrous environment
}

\author{
Albrecht Gerhard Meinel \\ Dupuytren-Ambulanz, Würzburg, Germany
}

Email address:

meinel_a@t-online.de

\section{To cite this article:}

Albrecht Gerhard Meinel. Dupuytren Contracture - How Fibromatosis Remodels the Palmar Subcutaneous Tissue and Its Fibrous Environment. Advances in Surgical Sciences. Vol. 1, No. 3, 2013, pp. 11-16. doi: 10.11648/j.ass.20130103.11

\begin{abstract}
Our understanding of the process of contracture of the fingers is based on extrinsic and intrinsic theories. Yet there has always been an inherent contradiction in these concepts. Additionally, nearly all of these theories proceed from the assumption of a contractio digitorum and this assumption is reflected in basic research as well. The author presents a concept, which describes the finger contracture more accurately as retention in the flexed position. This contraction-free concept, deduced from the function and structure of the palmar subcutaneous tissue, combines the different pathogenic aspects. Palmar fibromatosis primarily manifests itself in the palmar subcutaneous tissue. The function of this tissue was studied in the living hand; the anatomic structure of the subcutaneous fibrofatty tissue was studied for the first time in slice plastinates of adult hands. The palmar tissue exhibits varying tissue consistencies. It is compressed and expanded as the fingers move. In fibromatosis, fibrous nodules infiltrate the finger tissue in its shortened flexion configuration, which predominates both by day and by night. In the normal hand, the anchoring fibers allow nearly tension-free deformation of the skin tissue. In fibromatosis, this tissue loses its extensibility. Motion in the finger subjects the new formed tissue to tensile stress. This in turn provides the decisive stimulus for adaptive tissue transformation. This process does not require any active contraction. It plausibly explains the finger contracture as an extension block. In the contraction-free concept, the myofibroblast is understood to be a form of fibroblast that resists the tension arising in the tissue by isometric contraction. The contraction-free concept can explain all clinical pictures of fibromatosis as reactive remodeling of the specific local host tissue. It can also provide basic research with a conclusive anatomic pattern. Moreover, it implies a specific therapeutic paradigm: Treatment options that influence the formation of pathologic tissue and address the characteristic tensile stress will be able to control the root causes of the deformity.
\end{abstract}

Keywords: Dupuytren Disease, Palmar Fibro-Fatty Tissue, Flexion Configuration, Reactive Tissue Remodeling, Extension Block

\section{Introduction}

Dupuytren disease is classified as a benign tumor of connective tissue, a type of fibromatosis (WHO). This understanding has failed to gain widespread acceptance in clinical practice. The obvious changes in preexisting structures, particularly in the palmar aponeurosis, appear to support the widely accepted concept that the finger contracture is caused by "pathological shortening of the normal fascial structures [1]." On the one hand, there is the extrinsic concept of ingrowth of connective tissue into the palmar subcutaneous tissue. On the other hand, the changes to preexisting structures that characterize the clinical picture are indicative of an intrinsic process. Hueston [2] introduced the terms extrinsic and intrinsic into the discussion. Added to this is the image of a "contractio digitorum [3] ", which nearly all hypotheses have in common. No single consistent process model has yet been able to unify all of these aspects.

Dupuytren research today is primarily connective tissue research whose analysis of detail has shifted away from the hand. Research in cellular and molecular biology, biochemistry and genetics has led to some fascinating findings. Yet these findings can only be correctly interpreted and unified within the framework of an anatomic model: “... it is unlikely that histological appearance alone could lead to any further breakthrough in our understanding of the disease [4]." The purpose of this study is to clarify the structure and function of the palmar subcutaneous tissue, which the studies by Luck, 
MacCallum and Hueston, Thomine, Flint [5-8] and others have shown to be the primary tissue in which palmar fibromatosis manifests itself. One feels there should be some anatomic reference function in the normal hand that would provide a plausible model for deducing the development of the finger contracture in fibromatosis.

\section{What Was Studied}

The focus is on the palmar subcutaneous tissue of the hand. As Thomine indicated: "Finally the specific relationship between the skin and the deeper structures over the whole extent of the volar aspect of the hand should be borne in mind [7]."

Studies of the subcutaneous fibrous tissue are difficult because every open access, however minimally invasive, deranges the unstable fiber formation of the cutaneous retinacular system. It was only when von Hagens [9] produced slice plastinates of adult hands in the late 1970s that the framework for such studies improved dramatically. Until then there had been the fascinating sections of fetal hands by Stack [10] and Landsmeer [11]. However, these only allowed one to draw tentative conclusions about the anatomic site in the adult hand. The plastination technique made it possible to explore the skin-covered subcutaneous space using tissue slices of the complete site in the adult hand. Longitudinal and transverse tissue slices only a few millimeters thick were obtained from frozen hands with extended and flexed fingers and then preserved by plastination. Since then the results of these anatomic studies have been confirmed by over thirty years of work in office consultations and surgery.

\section{The Palmar Soft Tissue in the Normal Hand}

Most authors accept that palmar fibromatosis primarily manifests itself in the palmar tissue of the hand. The subcutis represents the palmar soft tissue, which in part is firmly attached to the underlying structures and in part remains mobile and flexible (Fig. 1).

The palmar tissue is generally thought to have a cushioning effect that improves the grip. Yet it is only in the fingertips that this cushioning is provided by compression-resistant tissue. In contrast, the finger tissue attached to the variable-length tendon sheaths is highly mobile. The finger tissue extends proximally to a four-finger line drawn through the two transverse palmar creases. In finger flexion, the palmar tissue is pressed upward from either side of the finger, pushed together, and folded up between the transverse skin creases. These deformations stop at the four-finger line. In finger extension, the finger tissue is shifted downward to either side of the finger, drawn apart, and unfolded. Over the mid-palm center between the thenar and hypothenar, the tissue is relatively firmly attached to the underlying aponeurosis (Fig. 1 and Fig. 3). The tissue of the mid-palm center and in the fingertips does not undergo deformation.

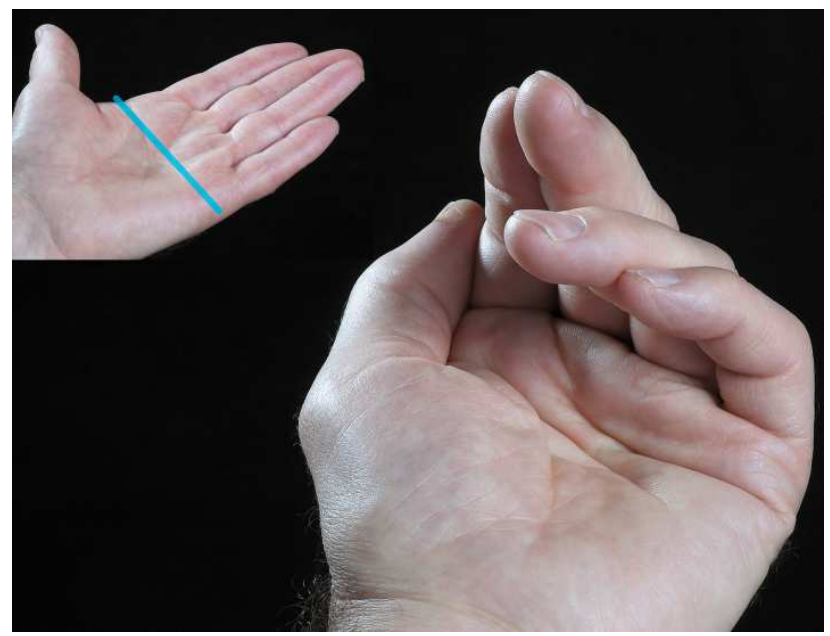

Figure 1. The contour of the palmar tissue in the living hand. Shortened flexion configuration and lengthened extension configuration. The four-finger line (blue) marks the proximal margin of the finger tissue. The flexed fingers represent the predominant posture of the fingers by day and by night.

The length of the tissue compartment in the fingers varies with their motion. The tendons leave the tissue compartment as it shortens and slide back into the compartment as it lengthens. The tendon sheaths are pushed together and drawn apart. The skin tissue undergoes smooth and apparently fluid deformation in the moving fingers.

In Fig. 1 the closing hand has purposely been placed in the foreground. This posture with flexed fingers is the predominant posture of the hand by day and by night. The shortened tissue configuration is the setting in which tissue remodeling in fibromatosis becomes established - not in the lengthened extension configuration. Dupuytren disease immobilizes the tissue in the shortened configuration of finger flexion. This in effect holds the finger in a flexed position. There is no basis for the assumption that a contraction process is at work.

The subcutaneous mesh of fibers acts as a cutaneous retinacular system. In the flexed finger, this mesh is pressed together into folds, shortened in its entirety, and exhibits a striking vertical parallel alignment. In the extended finger, the mesh is drawn apart, unfolded, and lacks any significant alignment of its fibers (Fig. 2). The tissue deformations appear as a smooth, continuous shift in the tissue between its extension and flexion configurations.

The mobile fiber components occur only over the fibrous tendon sheaths (Fig. 3). They exhibit over the entire length of the tendon sheaths the same configuration principle as the crisscrossed peritendinous skin-anchoring fibers. The fibers are embedded in fatty tissue. This fat acts not only as a barrier layer but also as a glide layer. The skin anchoring fibers arise from the sides of the tendon sheaths, course obliquely and transversely to the axis of the finger in a crisscrossed peritendinous pattern, and then insert into the skin. Grayson first described these fibers as "superficial or 
volar skin retinacula [12]" without recognizing their peritendinous crisscrossed configuration. There is a gap between the tendon sheaths and the subcutaneous tissue supported by the peritendinous crisscrossed fibers. The peritendinous crisscrossed fibers in the region of the natatory ligament are a lot longer. As they course to the skin, they bridge the interdigital spaces over a long distance.

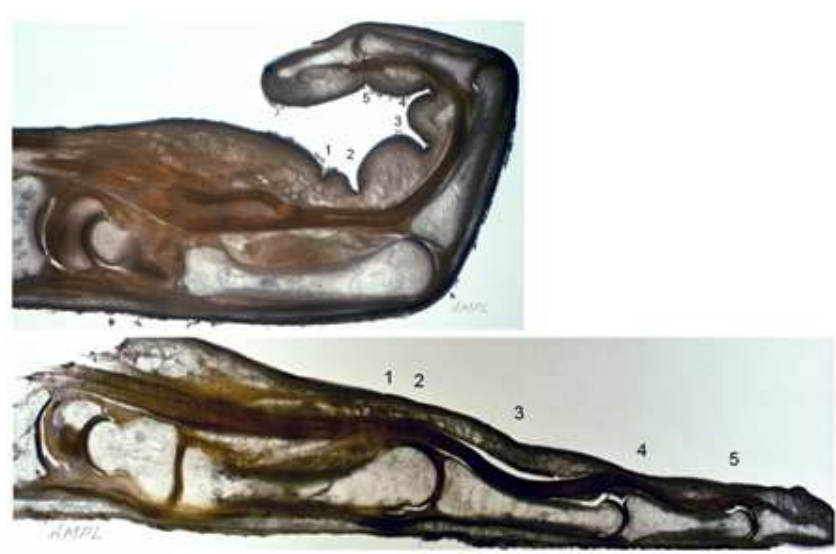

Figure 2. The palmar finger tissue in its varied configurations. Top: The palmar finger tissue in the flexion configuration (ring finger). Below: The palmar finger tissue in the extension configuration (middle finger). Slice plastinates of entire hands. 1 and 2 palmar creases; 3 palmar digital crease; 4 and 5 interphalangeal joint creases.
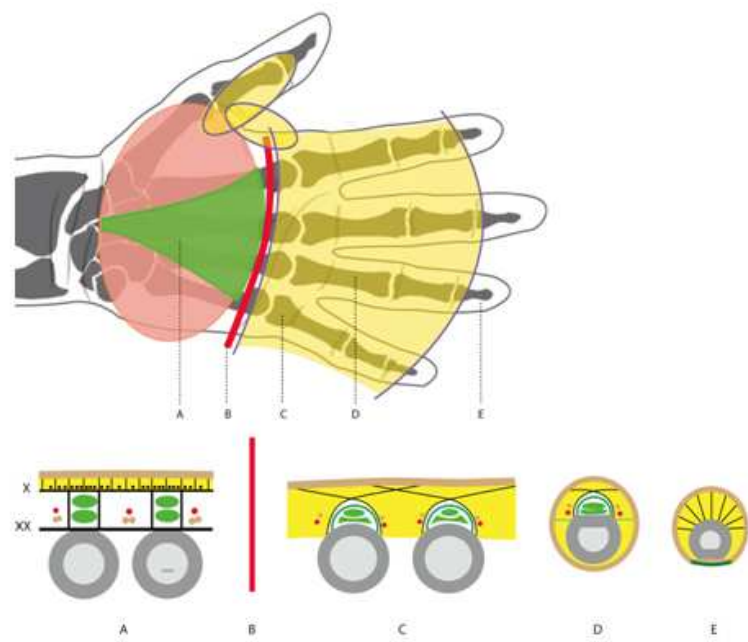

Figure 3. Topography and structure of the tissue consistencies in the palm. Top: Yellow: mobile finger tissue. Green: stationary tissue overlying the palmar aponeurosis in the mid-palm. Below: The structure of the cutaneous retinacular system. $A=$ the short mesh of fibers superficial to transverse and longitudinal fibers of the aponeurosis $(X)$, the vertical septa, and the transverse metacarpal ligament $(X X) ; B=$ four-finger line; $C=$ long peritendinous crisscrossed fibers in the region of the natatory ligament. $D$ = peritendinous crisscrossed fibers in the phalangeal segment; $E=$ radial fibers in the tissue of the fingertips.

There is no actual natatory ligament in the sense of a continuous transverse band. Along the sides of the finger, the Cleland fibers that connect the phalangeal bones to the skin define the border between the palmar and dorsal finger tissue. The peritendinous crisscrossed fibers are easily displaced and deflected within the fatty tissue. This ensures the passive tissue deformation that is crucial to unrestricted motion in the fingers. This passive deformation is the anatomic reference function we have been seeking. Its derangement impairs mobility in the finger and leads to an extension block.

\section{The Palmar Tissue in the Diseased Hand}

"The fibroblastic invasion and replacement of the palmar fat is regarded as the clue to the production of Dupuytren's tissue. The sequence can be followed from vascular invasion and perivascular cellular proliferation to maturing nodule and finally to a relatively acellular dense atrophic tendinous band [6]."

The palmar subcutaneous tissue is remodeled by infiltrating connective tissue (Fig. 4). The pericyte, a pluripotential mesenchymal cell of the capillary wall, is thought to be the mother cell. The infiltrating tissue displaces the fatty tissue. The fate of the initially fibroblastic nodule is largely determined by the interaction between host and tumor tissue. Nodular tissue within the stationary tissue overlying the palmar aponeurosis is not subjected to any tensile stress. It retains its nodular shape. However, where such tissue infiltrates the mobile subcutaneous tissue of the fingers, it may be assumed to do so primarily in the predominant shortened tissue configuration. Finger extension subjects the subcutaneous conglomerate to a tensile stress that influences decisively the histogenesis and morphogenesis of the Dupuytren tissue. The tensile stress transforms the proliferative hypercellular nodule into a hypocellular, collagen-rich cord. Adjacent and underlying structures, such as the aponeurosis, are also subjected to the tensile stress and become hypertrophied. The consistency of the tissue of the palm and its underlying structures changes. The palmar tissue loses its extensibility and begins to stiffen in the shortened configuration. The affected finger can no longer be freely extended, leading to the clinical picture of an extension block.

The process of tissue tethering in the flexion configuration is readily apparent in the beginning of the disease process. The nodule in the palm is not visible in the flexion configuration. The typical U-shaped fold contour appears only when the finger is extended. This picture is attributable to circumscribed tissue fixation in the flexion position and not to a contraction of the skin.

The skin anchoring fibers become entrapped in bundles by the infiltrating tissue. The greater the extent of this entrapment, the shorter the free length of the fibers and the lesser their mobility. This in turn restricts the mobility of the overlying skin anchored by these fibers. Amazingly, the entrapped fibers retain their filamentous structure [13]. 

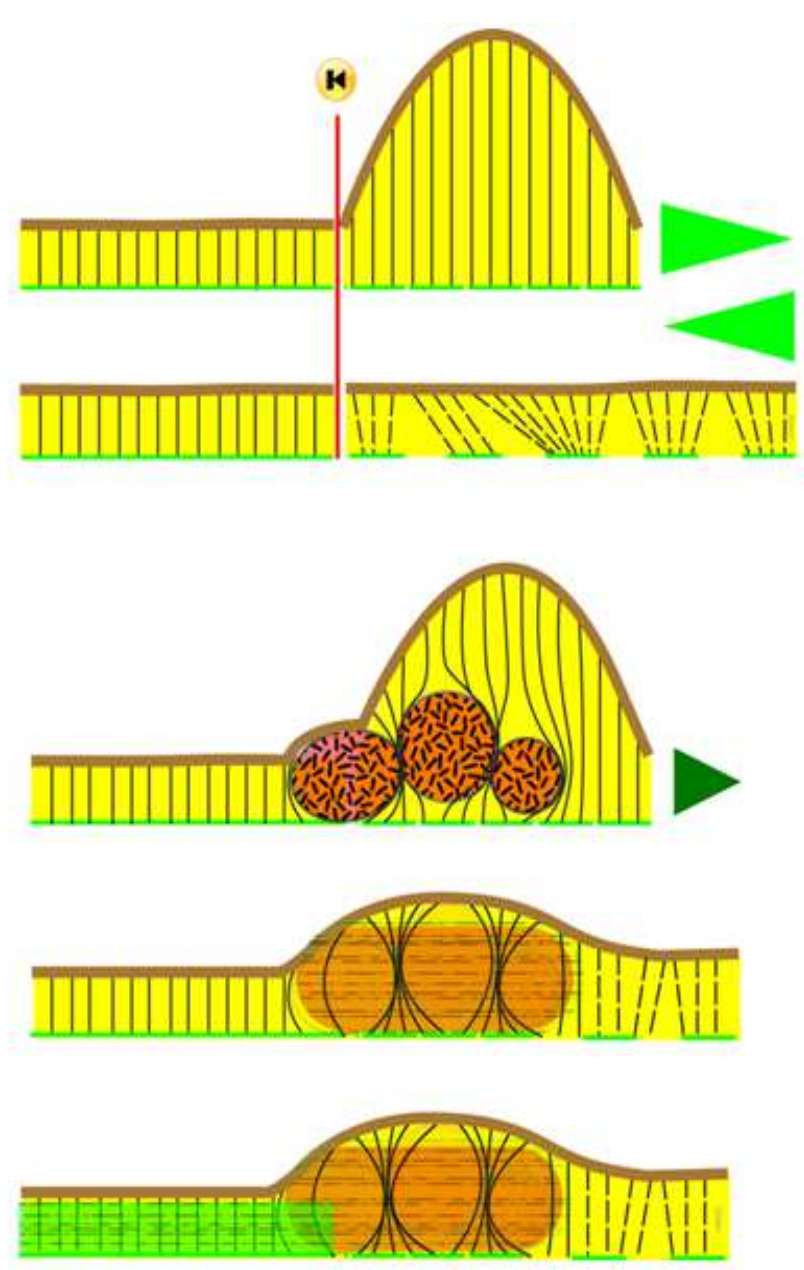

Figure 4. Interaction of tumor and host tissue

Top: Tissue deformation in the normal hand. The red line marks the border between stationary and flexible tissue. Below: Tissue infiltration and remodeling in palmar fibromatosis. The disorganized hypercellular proliferative tissue invades the subcutaneous tissue, entraps the skin anchoring fibers in bundles, and tethers the tissue of the palm to the finger tissue. Reactive remodeling of the nodular tissue to fibrous cord tissue with secondary hypertrophy of the tethered aponeurotic fibers (green bar). Loss of extensibility of the finger tissue.

A look at fibromatosis in regions other than palmar side of the hand lends support to contraction free theory. The specific morphologic and functional manifestation of superficial fibromatosis varies according to the location of its subcutaneous infiltration. The infiltrating fibrous tissue in the affected region of sole of the foot is not subjected to any tensile stress. This nodular tissue retains its nodular shape. In the shaft of the penis, the nodular tissue establishes itself in the shortened tissue configuration of the flaccid penis. In an erection, as in extension of the finger, the extensibility of the shaft tissue is impaired. The dorsal nodules overlying the proximal interphalangeal joints infiltrate the tissue in its lengthened configuration due to the predominance of finger flexion. This does not impair finger flexion.

\section{The Role of Myofibroblasts in the Tissue Remodeling Occurring in Fibromatosis}

The discovery of myofibroblasts [14] appears to confirm the old image of a contractio digitorum. The myofibroblast is seen as the driving force in a contraction that leads to tissue shortening. However, there are also good reasons for regarding myofibroblasts as the form of connective tissue cell that is simply responsible for connecting tissue together.

Myofibroblasts are not cells specific to palmar fibromatosis. As an intermediate cell form between the fibroblast and smooth muscle cell, the myofibroblast exhibits characteristics of both cells. The myofibroblast is a form of connective tissue cell whose primary function is "mechanical force production and collagen synthesis [15]." The force production of the myofibroblasts can of course lead to a contraction resulting in shortening. This is how the isolated cells appear in vitro experiments. In the tissue of the palm they appear to be the right cells, which resist tension arising in the tissue. This image is consistent with the findings of Hinz and Gabbiani who observed that the activities of the myofibroblasts are decisively influenced "by the constant mechanical feedback that the cells receive from the ECM. Stiffer ECM leads to higher myofibroblast contraction and ECM secretion ... [15] ".

Schultz and Tomasek described the contracting myofibroblast as a "tractofibroblast [16]" from the Latin tractare, to pull. I feel the term tractofibroblast more accurately describes the fibroblast form characteristic of Dupuytren disease when we derive the prefix from the Latin trahere, also to pull. Yet here it comes from the perfective form of the past passive participle tractum, meaning pulled. Accordingly, a tractofibroblast would be a fibroblast that has been pulled on. The myofibroblast in palmar fibromatosis is thus the fibroblast under tension or tractofibroblast, which immobilizes the finger in the flexed position.

The hand as an anatomic site lacks any analog of the picture of active tissue contraction such as occurs in the often cited contraction of the edges of a wound. In the diseased palmar subcutaneous tissue there are no "tissue extremities" that must be drawn together by contraction. In the soft tissue of the finger, infiltrating connective tissue solidifies a preexisting shortened tissue configuration which is then maintained against resistance by isometric cell contraction.

\section{Pathogenesis and Anatomy of the Extension Block}

The most obvious anatomic changes in the Dupuytren hand are seen in the cord structures that connect the palmar aponeurosis to the flexed fingers. There are other obvious changes, but these are only seen by the surgeon. Such 
changes include the thickened vertical septa of the palmar aponeurosis and the displacement of the proper palmar digital nerve and artery.

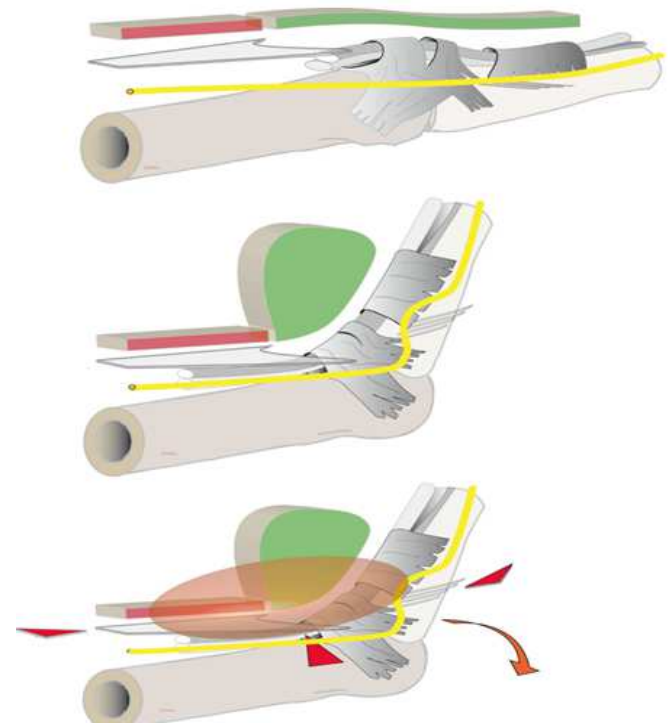

Figure 5: Pathogenesis and anatomy of the extension block.

Top and center: The tissue of the palm over a metacarpophalangeal joint in the normal hand. Green: the finger tissue superficial to the A1 and A2 pulleys. Brown: the tissue of the palm superficial to the bifurcating fibers of the aponeurosis. Yellow: proper palmar digital nerve. Below: In fibromatosis the tissues of the finger and palm become joined together. The tissue conglomerate is subjected to tensile stress which also acts on the deep structures attached to it: the aponeurosis, vertical septa, and tethered Cleland fibers (red arrows). The hypertrophied Cleland fibers become a "spiral cord." The apparent dystopic position of the neurovascular structures corresponds to their position in the flexed normal finger.

In Fig. 5, the tissue of the palm with its basic structures overlying a metacarpophalangeal joint is shown in the normal hand and the diseased hand. The close proximity of flexible and stationary palmar tissue corresponds to the deep structures, which in finger extension are drawn apart and in finger flexion are pushed close together in the bifurcation of the aponeurosis. In fibromatosis, infiltrating connective tissue leads to loss of extensibility of the palmar tissue. When this occurs, the tensile stress that builds up in this tissue in finger extension is also transferred to the basic structures, causing them to become hypertrophied. The figure illustrates how the palmar aponeurosis, the vertical septa and the Cleland fibers are among the deep structures that may show stress adaptation. The tumor tissue is transformed into tendon-like tissue aligned along the lines of stress, and the attached tissue structures become hypertrophied. Together these tissues produce the characteristic cords, which typify the clinical picture and block finger extension. The bowstring position of the Dupuytren tissue is consistent with the fibrotic remodeling of the tissue in its folded-up configuration.

The apparent dystopic position of the palmar nerve and artery in the Dupuytren finger in fact represents the tethering of these structures in their position in the flexed finger. The nerve and artery are unable to leave the shortening compartment in flexion. They are folded into loops above the raised Cleland fibers in a position that closely resembles intraoperative findings of palmar displacement. Some of the Cleland fibers may become attached to the Dupuytren tissue and become hypertrophied. This results in typical findings of cord tissue deep to the neurovascular bundles. This is generally described as the spiral cord even though it is not the cord itself but the nerve and artery that course in a spiral.

\section{Conclusion}

In attempting to gain a plausible understanding of the finger contracture by examining the fibrotic remodeling of the palmar subcutaneous tissue, it becomes apparent that the clinical picture is far more explainable as passive retention of the flexed finger than as active contraction of the extended position.

Luck was among the first to describe the histogenetic process in fibromatosis as a reaction to the infiltrating fibrous tissue and to draw the appropriate therapeutic conclusions. He classified "all the tissues identified with Dupuytren's contracture ... as the essential fibrous nodules, reactive tissues, and residual tissues [5]." What is new are the insights into the structure and function of the subcutaneous palmar tissue. What is new is the focus on the digital tissue overlying the variable-length tendon sheaths. What is also new is that the contraction piece has now been removed from the Dupuytren puzzle. Fibromatosis turns the tension-free tissue flexibility into a tension-generating tissue blockade. What is new, finally, is that the understanding of the process of fibromatosis now unifies extrinsic and intrinsic aspects. The infiltrating proliferative tissue represents the extrinsic process; the reacting host tissue represents the intrinsic process. Even the transformation of the fibroblast into a myofibroblast or tractofibroblast may be understood as a product of stress adaptation and not as a cause of contraction. Luck still retained the image of a contraction process in his concept. Yet he was the first to remove this process from the palmar aponeurosis and localize it in the newly infiltrating tissue. He understood this tissue to be reduced in volume due to involution that exerts a tensile stress on adjacent tissue, causing it to become hypertrophied. The image of an "involutional (contracting) stage" that Luck introduced "for descriptive purposes [5]" is more plausibly understood as stress adaptation. However, Luck's actual characterization of the tissue is complete as it stands and indeed is now more current than ever.

As long as we are unable to treat the underlying causes of the disease, the search for a process-oriented treatment strategy must have priority. This includes the recommendation first voiced by Luck [5] to refrain from resection of the hypertrophied aponeurosis. Limiting the fasciectomy to a digital, subcutaneous fasciectomy has proven very effective in my own patients. It also makes sense to ensure that the fibrotic remodeling takes place in 
the lengthened tissue of the extension configuration. This is the purpose of static extension splints worn as night splints. Objective verification of the efficacy of splinting is still lacking. However, its widespread use is indicative of a wealth of subjective clinical evidence. Splinting requires further, comprehensive evaluation.

Probably the most effective manner of influencing the process of fibromatosis is to apply measures that either remove the Dupuytren tissue's ability to react to tensile stress or remove the tensile stress itself. Cytology and biochemistry provide fascinating insights into the control mechanisms that allow connective tissue to respond to tensile stress with an adaptive cytologic reaction. Therefore the goals of basic research include one day being able to influence fibrotic remodeling by pharmocologic intervention in the cellular control mechanism. Yet even now surgeons have effective options for reducing or eliminating the mechanical tissue stress. These include the percutaneous needle fasciotomy as minimally invasive procedure, appropriate splinting, and the "no-tension program" espoused by Davis and Eaton [17]. The surgical options also have the advantage of focusing treatment on the abnormal tissue in the hand. Why should not an early needle fasciotomy and postoperative splinting succeed in delaying the development of severe hand deformations until advanced age or even halting it entirely?

The free unimpaired shifting of finger tissue is the reference function in the normal hand. The pathologic disorders arising from the derangement of this function can explain the morphologic and functional manifestations of Dupuytren disease. The fibrotic remodeling is initiated by infiltrating fibrous tissue. The fibrotic remodeling then is perpetuated by the stress-induced reactions of all involved connective tissue structures. This understanding of the process can explain all clinical pictures of superficial fibromatosis as specific local responses of the host tissue to the infiltration by the fibrous nodules that Luck describes as the "essential lesion [5]."

MacCallum and Hueston wrote in 1962: " . . . we conclude that, although the palmar aponeurosis is intimately involved in Dupuytren's contracture, this represents only one aspect of a change which may occur in any part of the network of palmar connective tissue and is usually secondary to changes arising within the fibrofatty tissue on its superficial aspect [6]." As we have shown: secondary to changes arising within the mobile fibrofatty tissue superficial to the fibrous tendon sheaths.

\section{Disclosure}

The author developed the concept of the Fixxglove night extension orthosis and is licenser for this product.

\section{References}

[1] McFarlane RM (1990) The finger. McFarlane RM et al (eds.) Dupuytren's disease, Churchill Livingstone Edinburgh London Melbourne New York:155-167
[2] Hueston JT (1985) Overview of aetiology and pathology. Hueston JT, Tubiana R (eds.) Dupuytren's disease, second edition G.E.M.Monograph, Churchill Livingstone Edinburgh London Melbourne New York:75-81

[3] Plater F (1614) Observationum, in hominis affectibus plerisque, corpori \& animo, functionum laesione, dolore, aliave molestia \& vitio incommodantibus. C. Waldkirch, Basileae: 140

[4] Shum DT (1990) Histopathology. McFarlane R M et al (eds.) Dupuytren's disease, Churchill Livingstone Edinburgh London Melbourne New York:25-30

[5] Luck JV (1959) Dupuytren's contracture: a new concept of the pathogenesis correlated with surgical management. J Bone Joint Surgery Am 41:635-664

[6] MacCallum P, Hueston JT (1962) The pathology of Dupuytren's contracture. Aust N Z J Surg 31:241-253

[7] Thomine JM (1985) The development and anatomy of the digital fascia. Hueston JT, Tubiana R (eds.) Dupuytren's disease, second edition G.E.M.Monograph Churchill Livingstone Edinburgh London Melbourne New York:3-12

[8] Flint MH (1990) Connective tissue biology. McFarlane RM et al (eds.) Dupuytren's disease, Churchill Livingstone Edinburgh London Melbourne New York:13-24

[9] von Hagens G (1979) Impregnation of soft biological specimens with thermosetting resins and elastomers. Anat Rec 194:247-255

[10] Stack HG (ed.) (1973) The palmar fascia. Churchill Livingstone Edinburgh London

[11] Landsmeer JMF (ed.) (1976) Atlas of anatomy of the hand. Churchill Livingstone Edinburgh London New York

[12] Grayson J (1940) The cutaneous ligaments of the digits. J Anatomy 75:164

[13] Meinel A (2012) Palmar fibromatosis or the loss of flexibility of the palmar finger tissue: A new insight into the disease process of Dupuytren contracture. C. Eaton et al. (eds.), Dupuytren's disease and related hyperproliferative disorders, Springer-Verlag Berlin Heidelberg:11-20

[14] Gabbiani G, Majno G (1972) Dupuytren's contracture: fibroblast contraction? An ultrastructural study. Am J Pathol $66: 131-146$

[15] Hinz B, Gabbiani G (2012) The role of the myofibroblast in Dupuytren's disease: Fundamental aspects. C. Eaton et al. (eds.), Dupuytren's disease and related hyperproliferative disorders, Springer-Verlag Berlin Heidelberg:53-60

[16] Schultz RJ, Tomasek JJ (1990) Cellular structure and interconnections. McFarlane RM et al (eds.) Dupuytren's disease, Churchill Livingstone Edinburgh London Melbourne New York:86-98

[17] Davis P, Eaton Ch (2012) Hand therapy for Dupuytren's contracture. C. Eaton et al. (eds.), Dupuytren's disease and related hyperproliferatve disorders, Springer-Verlag Berlin Heidelberg:305-316. 